\title{
Higher Order Weak Interactions and the Equivalence Principle
}

\author{
Ephraim Fischbach, Dennis E. Krause, and Carrick Talmadge \\ Physics Department, Purdue University, West Lafayette, IN 47907-1396 \\ Dubravko Tadić \\ Zavod za Teorijsku Fiziku, University of Zagreb, 41000 Zagreb, Croatia
}

(September 27, 1995)

\begin{abstract}
We consider the possibility that gravity may couple anomalously to the weak interaction and thereby lead to a violation of the Weak Equivalence Principle. It is shown that the contribution to the energy of a nucleus arising from neutrino-antineutrino exchange can be calculated rigorously, and may be the dominant contribution from higher order weak interactions. At the level of sensitivity that could be reached in the proposed STEP (Satellite Test of the Equivalence Principle) experiment, detection of an acceleration difference between the test masses could lead to new constraints on the coupling of gravity to neutrinos, and to higher-order weak interactions.
\end{abstract}

$\sin 9549$

To appear in the November 15th issue of Physical Review D 


\section{INTRODUCTION}

Recent interest in the possibility of deviations from the predictions of Newtonian gravity [1-5] has led to remarkable advances in the technology of experimental searches for violations of the Weak Equivalence Principle (WEP). This assumption, that the acceleration of an object in a gravitational field is independent of its chemical composition (universality of free fall) is central to both relativistic and non-relativistic theories of gravity. A breakdown of the universality of free fall (UFF) can arise in at least two distinct ways: a) A new long-range force coexisting with gravity (e.g. the so-called "fifth force" $[6,7]$ ) will in general produce such an effect. b) The gravitational interaction itself may fail to respect the WEP. It is the latter possibility that we wish to explore in the present paper, by focusing on possible deviations from the WEP arising from the higher-order weak interactions. We demonstrate that at the sensitivity level of the proposed STEP experiment [8] (Satellite Test of the Equivalence Principle) new and interesting limits will emerge on the coupling of gravity to weak interaction energy, and particularly to neutrinos. We show specifically that the contribution to the mass-energy of a nucleus arising from neutrino-antineutrino $(\nu \bar{\nu})$ exchange can be calculated rigorously, and may be the dominant higher-order weak contribution. Moreover, an anomalous coupling of gravity to the weak energy arising from $\nu \bar{\nu}$-exchange would have a characteristic composition-dependence, and this can be used to isolate this contribution and to thereby set limits on an anomalous coupling of gravity to neutrinos.

To frame more carefully the motivation for our work we briefly review the history of precision tests of the WEP and UFF. The experiments by Eötvös, Pekàr, and Fekete [9] were originally interpreted as supporting UFF and WEP to a precision $\Delta a / g \simeq 5 \times 10^{-9}$, where $\Delta a$ is the acceleration difference of the two samples being compared, $g=|-\vec{\nabla} U|$, and $U$ is the Newtonian potential. A subsequent reanalysis $[6,7]$ of the Eötvös experiment uncovered a systematic effect in the data which could be interpreted as a breakdown of UFF. This in turn helped to stimulate the recent revival of interest in such tests, as well as in 
theoretical models of how a breakdown of the WEP can come about [3].

Following the suggestion of a possible "fifth force" $[6,7]$, a large number of experiments have been carried out comparing the accelerations of different pairs of test materials to various sources. Modern experiments differ from the original Eötvös experiment not only in their design, but in their philosophy as well. Rather than study many pairs of materials, as Eötvös and collaborators did, recent experiments focus on comparing the acceleration of a single pair of materials, or at most a small number of such pairs [1-5]. This shift in philosophy can be traced to the classic experiment of Roll, Krotkov, and Dicke [10] and to a paper by Wapstra and Nijgh [11]. The shift stems from the realization that WEP cannot be valid unless gravity couples universally to all types of energy, since only a universal coupling can guarantee that samples of matter composed of different proportions of mass-energy, strong-energy, electromagnetic-energy, ..., will fall with identical accelerations. If gravity does couple anomalously to energy of type- $\alpha$ ( $\alpha=$ strong, electromagnetic, weak, ...), then a sample's type- $\alpha$ energy content contributes differently to its inertial and (passive) gravitational masses. One finds that the gravitational mass of a sample $i,\left(m_{G}\right)_{i}$, can be expressed as,

$$
\left(m_{G}\right)_{i}=\left(m_{I}\right)_{i}+\eta_{\alpha}\left(E_{\alpha}\right)_{i} / c^{2}
$$

where $\left(m_{I}\right)_{i}$ and $\left(E_{\alpha}\right)_{i}$ are the sample's inertial mass and type- $\alpha$ energy content, and where $\eta_{\alpha}$ is a dimensionless constant whose magnitude reflects the strength of the violation of the weak equivalence principle induced by the anomalous coupling to type- $\alpha$ energy. Since the gravitational acceleration of sample $i$ is $a_{i}=\left(m_{G}\right)_{i} g /\left(m_{I}\right)_{i}$, an experiment like that of Roll, Krotkov, and Dicke sets a limit on the difference between the accelerations of a selected pair of samples,

$$
\frac{a_{1}-a_{2}}{g}=\eta_{\alpha}\left[\left(\frac{E_{\alpha}}{m_{I} c^{2}}\right)_{1}-\left(\frac{E_{\alpha}}{m_{I} c^{2}}\right)_{2}\right] .
$$

When combined with estimates of the type- $\alpha$ energy content of each sample, this experimental limit implies a constraint on the magnitude of the parameter $\eta_{\alpha}$, and hence, on the nature of the coupling between gravity and matter. 
Wapstra and Nijgh [11] used the semi-empirical mass formula [12,13] to estimate the nuclear and electromagnetic energy content of such samples. Recently, more detailed estimates of the energy content of samples have been used to refine the interpretation of WEP tests [14]. The first estimate of the weak contribution was made by Nordtvedt [15], and this was followed by a more detailed analysis by Haugan and Will [16], who calculated the contribution of the parity-conserving (pc) weak interaction to the ground state state energies of nuclei. They concluded that $\left|\eta_{p c}\right| \lesssim 0.01$ and, thus, that parity-conserving-weak interaction energies obey the equivalence principle to better than a part in 100. Fischbach et al. [17] lent support of this constraint by showing that an equivalence-principle-violating coupling to the intermediate vector bosons studied by Nielsen and Picek [18] leads directly to Eq. (1.2).

The preceding discussion can be extended by observing that WEP experiments also imply limits on possible anomalous direct couplings of gravity to various elementary particles. We note that in a field theory description, a nucleus has a nonzero probability amplitude for containing particle-antiparticle pairs. It follows that the interaction of a nucleus with the gravitational field probes the separate interactions of these particles with gravity. Schiff [19] started from such a picture to set a limit on the possibility that matter and antimatter "fell" in opposite directions in a gravitational field. As we see from Fig. 1, the amplitude for producing a virtual $e^{+}-e^{-}$pair in the field of a nucleus of charge $Z$ is proportional to $\left(Z e^{2}\right)^{2}$, where $e$ is the unit of electric charge. Hence the contribution $E_{\text {pair }}$ from such a diagram to the gravitational mass-energy of a nucleus is of order

$$
E_{\text {pair }} \simeq m_{e} c^{2}(Z \alpha)^{2}
$$

where $\alpha=e^{2} / \hbar c$ and $m_{e}$ is the electron (or positron) mass. By combining Eqs. (1.1)-(1.3) a limit on a possible anomalous coupling of gravity to antimatter can be inferred. This limit is of interest in connection with current attempts to directly compare the acceleration of matter and antimatter [20]. The Schiff argument is particularly interesting because of the possibility that it could be taken over, with the obvious modifications, to set limits on possible anomalous couplings to neutrinos and antineutrinos. 
The focus of the present paper is the $\nu \bar{\nu}$-exchange contribution to the mass-energy of a nucleus shown in Fig. 2, which can be viewed as the direct weak interaction analog of Fig. 1. We will show that not only can this contribution be calculated rigorously, but it is likely the dominant contribution in $O\left(G_{F}^{2}\right)$, where [21] $G_{F}=1.16639(2) \times 10^{-5} \mathrm{GeV}^{-2}(\hbar c)^{3}$ is the Fermi decay constant. The first correct calculation of the 2-body potential arising from Fig. 2 was carried out by Feinberg and Sucher [22] who used an effective low-energy 4-fermion interaction involving only charged currents. They found for the potential energy $V_{e e}^{(2)}(r)$ describing the interaction of two electrons via the $\nu \bar{\nu}$-exchange diagram shown in Fig. 2,

$$
V_{e e}^{(2)}(r)=G_{F}^{2} / 4 \pi^{3} r^{5}
$$

where $r=\left|\vec{r}_{1}-\vec{r}_{2}\right|$ is the separation of the electrons. (Except where otherwise noted, we set $\hbar=c=1$.) Subsequently Feinberg, Sucher, and Au (FSA) [23] recalculated $V_{e e}^{(2)}(r)$ in the framework of the Standard Model and found

$$
V_{e e}^{(2)}(r)=G_{F}^{2}\left(2 \sin ^{2} \theta_{W}+1 / 2\right)^{2} / 4 \pi^{3} r^{5}
$$

where $\theta_{W}$ is the weak mixing angle, with [21] $\sin ^{2} \theta_{W}=0.2319(5)$. The result in Eq. (1.5) has been confirmed recently by Hsu and Sikivie [24] using a different formalism from FSA. In Sec. II we rederive Eq. (1.5) using the formalism of Schwinger [25] and Hartle [26,27], which lends itself naturally to a study of the many-body neutrino-exchange potentials. Owing to the fact that neutrino-exchange gives rise to long-range forces, the many-body potentials could lead to observable effects, as we discuss in greater detail below. In Sec. III we derive the expression for the 2-body contribution $W^{(2)}$ to the weak energy $W$ of a nucleus arising from neutrino exchange. Particular attention is devoted to analyzing the characteristic composition dependence of $W^{(2)}$, which is also a consequence of the long-range nature of the neutrino-exchange force. We also calculate the 4-body contribution $W^{(4)}$ and verify that it is small compared to $W^{(2)}$, as expected naively. We demonstrate, however, that many-body neutrino-exchange effects could be large in other systems, such as neutron stars or white dwarfs, and the implications of this are discussed. Our results are summarized in Sec. IV. 


\section{THE TWO-BODY NEUTRINO-EXCHANGE POTENTIAL}

The exchange of $\nu \bar{\nu}$ pairs among neutrons $(n)$ and protons $(p)$ in a nucleus gives rise to 2-body $n-n, p-p$, and $n-p$ potentials. Because of the long range of the neutrino-exchange force, interactions involving electrons should also be considered, but these are relatively smaller as we discuss in Sec. III. For the sake of definiteness we consider the $n$ - $n$ potential which will emerge as the dominant contribution. Following an argument due to Fermi [28] we note that the effects of a long-range force can be approximated by the classical (spinindependent) contribution which increases approximately as $N^{2}$, where $N$ is the number of neutrons. The $\nu \bar{\nu}$-exchange potential between neutrons, $V_{n n}^{(2)}(r)$ is thus analogous to the 2-body Coulomb potential

$$
V_{C}=e^{2} / r
$$

which leads directly to the familiar expression for the Coulomb energy $W_{C}$ of a nucleus with radius $R$ containing $Z$ protons $[12,13]$ :

$$
W_{C}=\frac{3}{5} Z(Z-1) \frac{e^{2}}{R}
$$

In fact we demonstrate explicitly in Sec. III below that $W_{C}$ and the 2-body weak contribution $W^{(2)}$ given in Eq. (3.9) can be derived using the identical formalism.

Following references $[22,23,26,27]$ we assume that the low energy neutrino-neutron coupling can be written in the form

$$
\begin{gathered}
\mathcal{L}_{I}^{\nu n}(x)=\frac{G_{F}}{\sqrt{2}} a_{n} \ell_{\mu}(x) N_{\mu}(x) \\
\ell_{\mu}=i \bar{\psi}(x) \gamma_{\mu}\left(1+\gamma_{5}\right) \psi(x) .
\end{gathered}
$$

Here $\psi(x)$ is the neutrino field operator, $\ell_{\mu}(x)$ is the neutrino current, $a_{n}$ is the neutrinoneutron coupling constant, and $N_{\mu}=i \Psi(x) \gamma_{\mu} \Psi(x)$ is the vector current for the neutrons, which is the only component which contributes to the static spin-independent 2-body potential. In the Standard Model the constants $a_{n}, a_{p}$, and $a_{e}$, which describe the coupling of neutrinos to neutrons, protons, and electrons respectively, are given by [21] 


$$
\begin{aligned}
& a_{n}=-\frac{1}{2} \\
& a_{p}=\frac{1}{2}-2 \sin ^{2} \theta_{W}=0.036 \\
& a_{e}=\frac{1}{2}+2 \sin ^{2} \theta_{W}=0.964
\end{aligned}
$$

Using Eqs. (2.3) and (2.4), the weak energy $W$ arising from neutrino-exchange among neutrons can be expressed in terms of the Schwinger formula [25-27]:

$$
W=\frac{i}{2 \pi} \operatorname{Tr}\left\{\int_{-\infty}^{\infty} d E \ln \left[1+\frac{G_{F} a_{n}}{\sqrt{2}} N_{\mu} \gamma_{\mu}\left(1+\gamma_{5}\right) S_{F}^{(0)}(E)\right]\right\}
$$

Here $S_{F}^{(0)}$ is the operator whose matrix element in configuration space, $S_{F}^{(0)}\left(\vec{x}, \vec{x}^{\prime}, E\right)$, is the Fourier transform of the usual free Feynman propagator $S_{F}^{(0)}\left(\vec{x}, \vec{x}^{\prime}\right)$ :

$$
S_{F}\left(x, x^{\prime}\right)=\int_{-\infty}^{\infty} \frac{d E}{2 \pi} e^{-i E\left(t-t^{\prime}\right)} S_{F}\left(\vec{x}, \vec{x}^{\prime}, E\right) .
$$

In our metric conventions $[29-31], S_{F}^{(0)}(\vec{x}, 0, E) \equiv S_{F}^{(0)}(\vec{x}, E)$ is given by

$$
S_{F}^{(0)}(\vec{x}, E)=\gamma \cdot \eta\left[\frac{i}{4 \pi} \frac{e^{i|E|(|\vec{x}|+i \epsilon)}}{|\vec{x}|+i \epsilon}\right] \equiv \gamma \cdot \eta \Delta_{F}(\vec{x}, E),
$$

where

$$
\gamma \cdot \eta=\vec{\gamma} \cdot \vec{\partial}-\gamma_{4} E
$$

As usual, the trace (Tr) in Eq. (2.5) is then understood as denoting both an integral in configuration space and a trace over Dirac indices.

Expanding Eq. (2.5) to $O\left(G_{F}^{2}\right)$, the 2-body energy $W^{(2)}$ is given by

$$
\begin{aligned}
W^{(2)}= & \frac{-i}{2 \pi}\left(\frac{G_{F} a_{n}}{\sqrt{2}}\right)^{2} \int d^{3} x_{1} d^{3} x_{2} \int_{-\infty}^{\infty} d E \\
& \times \operatorname{tr}\left\{\gamma_{\mu}\left(1+\gamma_{5}\right) S_{F}^{(0)}\left(\vec{r}_{12}, E\right) \gamma_{\nu}\left(1+\gamma_{5}\right) S_{F}^{(0)}\left(\vec{r}_{21}, E\right)\right\} N_{\mu}\left(x_{1}\right) N_{\nu}\left(x_{2}\right)
\end{aligned}
$$

Here $\vec{r}_{12}=\left(\vec{x}_{1}-\vec{x}_{2}\right), N_{\mu}\left(x_{1}\right)$ and $N_{\nu}\left(x_{2}\right)$ denote the external neutron currents at $x_{1}$ and $x_{2}$, and $S_{F}^{(0)}\left(\vec{r}_{i j}, E\right)$ is given by Eq. (2.7) with $\vec{x} \rightarrow \vec{r}_{i j}$ and $\eta \rightarrow \eta(i j)$. The overall minus sign arises from the expansion of $\ln [1+\cdots]$ in Eq. (2.5), and tr denotes the trace over the Dirac matrices in $\{\cdots\}$. The factors of $\left(1+\gamma_{5}\right)$ can be anticommutated past $S_{F}^{(0)}$ and, using the relation 


$$
\left(1+\gamma_{5}\right)^{2}=2\left(1+\gamma_{5}\right)
$$

we have

$$
\operatorname{tr}\{\cdots\}=2 \operatorname{tr}\left\{S_{F}^{(0)}\left(\vec{r}_{21}, E\right) \gamma_{\mu} S_{F}^{(0)}\left(\vec{r}_{12}, E\right) \gamma_{\nu}\left(1+\gamma_{5}\right)\right\}
$$

Since we are interested in computing the self energy of a static collection of neutrons (which are assumed to have no net polarization), the neutron currents are given in our metric conventions by [29-31] by,

$$
N_{\mu}\left(x_{1}\right)=i \rho_{1} \delta_{\mu 4} ; \quad N_{\nu}\left(x_{2}\right)=i \rho_{2} \delta_{\nu 4}
$$

where $\rho_{1}=\rho_{2}=\rho$ is the number density of neutrons. Combining Eqs. (2.11) and (2.12) we see that the term containing $\gamma_{5}$, whose trace is proportional to $\epsilon_{\mu \nu \lambda \rho}$, makes no contribution. The remaining terms give

$$
\begin{aligned}
W^{(2)}= & \frac{-(i)^{3}}{\pi}\left(\frac{G_{F} a_{n}}{\sqrt{2}}\right)^{2} \int \rho_{1} d^{3} x_{1} \int \rho_{2} d^{3} x_{2} \int_{-\infty}^{\infty} d E \\
& \times \operatorname{tr}\left[\gamma_{\alpha} \gamma_{4} \gamma_{\beta} \gamma_{4}\right] \eta_{\alpha}(21) \Delta_{F}\left(\vec{r}_{21}, E\right) \eta_{\beta}(12) \Delta_{F}\left(\vec{r}_{12}, E\right) .
\end{aligned}
$$

Using

$$
\operatorname{tr}\left[\gamma_{\alpha} \gamma_{4} \gamma_{\beta} \gamma_{4}\right]=4\left(2 \delta_{\alpha 4} \delta_{\beta 4}-\delta_{\alpha \beta}\right)
$$

the expression in curly brackets in Eq. (2.11) can be written as

$$
\begin{aligned}
\{(2.11)\} & =4\left[2 \eta_{4}(21) \eta_{4}(12)-\eta(21) \cdot \eta(12)\right] \\
& =4\left[E^{2}-\vec{\partial}_{12} \cdot \vec{\partial}_{21}\right]
\end{aligned}
$$

where $\vec{\partial}_{12} \equiv \partial / \partial \vec{r}_{12}$. Combining $(2.15)$ and $(2.13)$ we have

$$
\begin{aligned}
W^{(2)}= & \frac{4 i}{\pi}\left(\frac{G_{F} a_{n}}{\sqrt{2}}\right)^{2} \int \rho_{1} d^{3} x_{1} \int \rho_{2} d^{3} x_{2} \int_{-\infty}^{\infty} d E \\
& \times\left\{E^{2} \Delta_{F}\left(\vec{r}_{21}, E\right) \Delta_{F}\left(\vec{r}_{12}, E\right)-\vec{\partial}_{12} \cdot \vec{\partial}_{21} \Delta_{F}\left(\vec{r}_{21}, E\right) \Delta_{F}\left(\vec{r}_{12}, E\right)\right\} \\
= & \frac{4 i}{\pi}\left(\frac{G_{F} a_{n}}{\sqrt{2}}\right)^{2} \int \rho_{1} d^{3} x_{1} \int \rho_{2} d^{3} x_{2} \int_{-\infty}^{\infty} d E \\
& \times\left(\frac{i}{4 \pi}\right)^{2}\left\{E^{2} \frac{e^{i|E|\left(r_{12}+r_{21}+i \epsilon\right)}}{r_{12} r_{21}}-\vec{\partial}_{12} \cdot \vec{\partial}_{21}\left(\frac{e^{i|E|\left(r_{12}+r_{21}+i \epsilon\right)}}{r_{12} r_{21}}\right)\right\} .
\end{aligned}
$$


We note from Eqs. (2.7) and (2.8) that the operators $\vec{\partial}_{12}$ and $\vec{\partial}_{21}$ act on the respective coordinates $\vec{r}_{12}$ and $\vec{r}_{21}$ as if these were independent, notwithstanding the fact that $\vec{r}_{12}+\vec{r}_{21}=$ 0. This applies as well to all the derivative terms that appear in the many-body amplitudes.

Following Hartle [27] the integral over $E$ can be evaluated by considering the functions $\bar{I}_{n}(z)$ defined by

$$
\begin{aligned}
\bar{I}_{n}(z) & =\int_{-\infty}^{\infty} d E E^{n} e^{i|E|(z+i \epsilon)} \\
& = \begin{cases}2 \int_{0}^{\infty} d E E^{n} e^{i|E|(z+i \epsilon)} \equiv I_{n}(z) & \text { even } n \\
0 & \text { odd } n\end{cases}
\end{aligned}
$$

where $z=r_{12}+r_{21}$. Since $|E|$ is an even function of $E, \bar{I}_{n}(z)$ is nonzero only for even values of $n$. An elementary integration gives

$$
I_{0}(z)=\frac{2 i}{z+i \epsilon}
$$

and differentiating Eqs. (2.17) and (2.18) with respect to $z$ leads to

$$
-i \frac{d I_{0}(z)}{d z}=I_{1}(z)=\frac{-2}{(z+i \epsilon)^{2}} \text {. }
$$

Continuing in this way we find [27]

$$
I_{n}(z)=\frac{2 i^{n+1} n !}{(z+i \epsilon)^{n+1}}
$$

Combining Eqs. (2.16) and (2.20) allows $W^{(2)}$ to be written as

$$
\begin{aligned}
W^{(2)}= & \int \rho_{1} d^{3} x_{1} \int \rho_{2} d^{3} x_{2}\left\{\left(\frac{-1}{\pi^{3}}\right)\left(\frac{G_{F} a_{n}}{\sqrt{2}}\right)^{2}\right. \\
& \left.\times\left[\frac{1}{r_{12} r_{21}\left(r_{12}+r_{21}\right)^{3}}+\frac{1}{2} \vec{\partial}_{12} \cdot \vec{\partial}_{21} \frac{1}{r_{12} r_{21}\left(r_{12}+r_{21}\right)}\right]\right\} .
\end{aligned}
$$

The quantity in curly brackets in Eq. (2.21) is, evidently, the 2-body potential $V^{(2)}\left(r_{12}\right)$,

$$
V^{(2)}\left(r_{12}\right)=\frac{-1}{\pi^{3}}\left(\frac{G_{F} a_{n}}{\sqrt{2}}\right)^{2}\left[\frac{1}{r_{12} r_{21}\left(r_{12}+r_{21}\right)^{3}}+\frac{1}{2} \vec{\partial}_{12} \cdot \vec{\partial}_{21} \frac{1}{r_{12} r_{21}\left(r_{12}+r_{21}\right)}\right] \text {. }
$$

Since $r \equiv r_{12}=r_{21}$, first term in square brackets in Eq. (2.22) reduces to $1 / 8 r^{5}$. In the second term we note that the gradients act on a function which depends only on $r_{12}$ and $r_{21}$, and hence we can write 


$$
\vec{\partial}_{12} \cdot \vec{\partial}_{21}=\left(\hat{r}_{12} \frac{\partial}{\partial r_{12}}\right) \cdot\left(\hat{r}_{21} \frac{\partial}{\partial r_{21}}\right)=\hat{r}_{12} \cdot \hat{r}_{21} \frac{\partial}{\partial r_{12}} \frac{\partial}{\partial r_{21}}=-\frac{\partial}{\partial r_{12}} \frac{\partial}{\partial r_{21}}
$$

Using Eq. (2.23) the expression in square brackets in Eq. (2.22) can be written as

$$
[\text { Eq. }(2.22)]=\frac{1}{8 r^{5}}-\frac{5}{8 r^{5}}=-\frac{1}{2 r^{5}}
$$

and hence,

$$
V^{(2)}(r)=+\frac{G_{F}^{2} a_{n}^{2}}{4 \pi^{3}} \frac{1}{r^{5}} \equiv \frac{\kappa}{r^{5}}
$$

Eq. (2.25) gives the original FS result [22-24] when we set $a_{n}=1$, which is the value appropriate to the charged-current model of the weak interaction assumed by FS. We note that Eq. (2.25) applies separately to the exchange of each of the species $\nu_{e} \bar{\nu}_{e}, \nu_{\mu} \bar{\nu}_{\mu}$, and $\nu_{\tau} \bar{\nu}_{\tau}$.

For later purposes it is interesting to note that the functional form of $V^{(2)}(r)$ can be inferred on dimensional grounds, as noted originally by Feinberg and Sucher [22]. The only dimensional quantities upon which a static neutrino-exchange potential can depend are $G_{F}, r$ and (possibly) the masses of the external particles. However, in the non-relativistic limit appropriate to a static potential, bilinear covariants such as $\bar{u}\left(p^{\prime}\right) \gamma_{\lambda}\left(1+\gamma_{5}\right) u(p)$. are independent of the mass of the fermion characterized by the spinor $u(p)$. Thus the only relevant dimensional parameters are $G_{F}$ and $r$ and, since the 2-body operator is proportional to $G_{F}^{2}$, it follows that $V^{(2)}(r) \propto G_{F}^{2} / r^{5}$. Implicit in this argument is the assumption that no other dimensional parameters are present and, since the standard model is renormalizable, this will indeed be the case. This argument holds even in the framework of the (nonrenormalizable) charged-current model originally assumed by FS, since the regularization procedure employed by FS to extract the long-distance behavior of $V^{(2)}(r)$ introduces no additional mass parameters. 


\section{EVALUATION OF $W^{(2)}$}

\section{A. General Formalism}

From Eq. (2.21) the 2-body $\nu \bar{\nu}$-exchange contribution $W^{(2)}$ can be obtained by carrying out the integrals over $\vec{x}_{1}$ and $\vec{x}_{2}$ after writing $r=r_{12}=\left|\vec{x}_{1}-\vec{x}_{2}\right|$. The evaluation of these integrals can be simplified by considering the function $\mathcal{P}(r)$ which gives the normalized probability density for finding two points randomly chosen in a sphere to be a distance $r=r_{12}$ apart. The functional form of $\mathcal{P}(r)$ has been obtained by a number of authors $[32-34]$ and is given by

$$
\begin{aligned}
\mathcal{P}(r) & =\frac{3 r^{2}}{R^{3}}-\frac{9}{4} \frac{r^{3}}{R^{4}}+\frac{3}{16} \frac{r^{5}}{R^{6}} \\
& =\frac{3 r^{2}}{R^{3}}\left[1-\frac{3}{2}\left(\frac{r}{2 R}\right)+\frac{1}{2}\left(\frac{r}{2 R}\right)^{3}\right] .
\end{aligned}
$$

The average value $\langle g\rangle$ of any function $g(r)$ taken over a spherical volume is then given by

$$
\langle g\rangle=\int_{0}^{2 R} d r \mathcal{P}(r) g(r)
$$

where

$$
\int_{0}^{2 R} d r \mathcal{P}(r)=1
$$

Returning to the Coloumb problem we wish to calculate $\left\langle e^{2} / r\right\rangle$ by this method. From Eq. (3.1),

$$
e^{2}\langle 1 / r\rangle=e^{2} \int_{0}^{2 R} d r\left(\frac{3 r^{2}}{R^{3}}-\frac{9 r^{3}}{4 R^{4}}+\frac{3 r^{5}}{16 R^{6}}\right) \frac{1}{r}=\frac{6}{5} \frac{e^{2}}{R}
$$

The result in Eq. (3.4) gives the Coulomb energy for a single pair of charges spread out through a spherical volume, and for a nucleus containing $Z$ charges there are

$$
\left(\begin{array}{l}
Z \\
2
\end{array}\right)=\frac{1}{2} Z(Z-1)
$$

such pairs. Hence the final expression for the Coloumb energy $W_{C}$ is given by 


$$
W_{C}=\frac{1}{2} Z(Z-1) \frac{6}{5} \frac{e^{2}}{R}=\frac{3}{5} Z(Z-1) \frac{e^{2}}{R}
$$

in agreement with Eq. (2.2).

The $\nu \bar{\nu}$-exchange contribution can be calculated in an identical manner, the only difference being that the nucleon-nucleon hard core radius $r_{c}$ must be included explicitly. We find for $\left\langle V^{(2)}(r)\right\rangle$,

$$
\begin{aligned}
\left\langle V^{(2)}(r)\right\rangle & =\int_{\tau_{c}}^{2 R} d r \mathcal{P}(r) V^{(2)}(r) \\
& =\int_{r_{c}}^{2 R} d r \mathcal{P}(r)\left(\frac{\kappa}{r^{5}}\right) \\
& =\frac{3 \kappa}{2 R^{3} r_{c}^{2}} \xi(x),
\end{aligned}
$$

where

$$
\xi(x)=1-3 x+3 x^{2}-x^{3} ; \quad x=r_{c} / 2 R
$$

In analogy to the Coulomb case, the final expression for $W^{(2)}$ is then obtained by multiplying the result in Eq. (3.7) by $N(N-1) / 2$ which gives

$$
W^{(2)}=\frac{3}{4} \frac{\kappa N(N-1)}{R^{3} r_{c}^{2}} \xi(x)=\frac{3}{16 \pi^{3}} \frac{\left(G_{F} a_{n}\right)^{2}}{\hbar c} \frac{N(N-1)}{R^{3} r_{c}^{2}} \xi(x),
$$

where a factor of $\hbar c$ has been reinstated. Eq. (3.9) gives the desired expression for the contribution to $W^{(2)}$ from the $n-n \nu \bar{\nu}$-exchange potential. Evidently the $p-p$ and $n-p$ contributions can be obtained from Eq. (3.9) by replacing $a_{n}^{2}$ by $a_{p}^{2}$ or $a_{n} a_{p}$ respectively, and at the same time replacing $N(N-1) / 2$ by the appropriate combinatoric factor. For $p$ - $p$ this factor is $Z(Z-1) / 2$ while for $n-p$ it is $N Z$. Hence the contributions of $p-p$ and $n-p$ relative to $n-p$ are given by

$$
\begin{gathered}
\frac{W^{(2)}(p-p)}{W^{(2)}(n-n)}=\frac{a_{p}^{2}}{a_{n}^{2}} \frac{Z(Z-1)}{N(N-1)}=5.2 \times 10^{-3} \frac{Z(Z-1)}{N(N-1)} \\
\frac{W^{(2)}(n-p)}{W^{(2)}(n-n)}=\frac{a_{p}}{a_{n}} \frac{2 Z}{N-1}=-0.145 \frac{Z}{N-1}
\end{gathered}
$$


Since $Z \leq N$ for almost all nuclei it follows from Eq. (3.10) that $W^{(2)}(p-p)$ is negligible compared to $W^{(2)}(n-n)$. Similarly $\left|W^{(2)}(n-p)\right|$, although not negligible, is nonetheless small. Hence for practical purposes we can approximate the nuclear contribution to $W^{(2)}$ by the $n$ - $n$ contribution. Turning to the contributions from the $n-e$ potential, we note that since electrons are spread out through a volume of order $(1 \AA)^{3}$, where as nucleons are confined to a volume of order $(1 \mathrm{fm})^{3}$, it follows from Eq. (3.9) that contributions from electrons are suppressed relative to the $n-n$ contribution by a factor of order $(1 \mathrm{fm} / 1 \AA)^{3}=10^{-15}$. We conclude that among all possible interactions involving $n, p$, and $e$, neutrino-exchange between neutrons is the dominant contribution, and is given by the result in Eq. (3.9).

\section{B. Numerical Results}

We proceed to evaluate $W^{(2)}$ for a nucleus with $N$ neutrons and $Z$ protons, with $N+Z=$ $B$. The hard-core radius $r_{c}$ is taken to be [35],

$$
r_{c}=0.49 \times 10^{-13} \mathrm{~cm}
$$

and for $R$ we use [36]

$$
R \simeq 1.24 \times 10^{-13} \mathrm{~cm} \cdot B^{1 / 3}
$$

The function $\xi(x)$ in Eq. (3.8) now becomes a function of $B$ given by

$$
\xi(B)=1-\frac{0.593}{B^{1 / 3}}+\frac{0.117}{B^{2 / 3}}-\frac{0.008}{B} .
$$

$W^{(2)}$ can then be expressed in the form

$$
W^{(2)}=1.34 \times 10^{-7} \mathrm{eV} \frac{N(N-1)}{B} \xi(B) .
$$

Since $W^{(2)}$ represents the energy $E_{\alpha}$ in Eq. (1.1) arising from neutrino exchange, it follows from Eq. (1.2) that the quantity which determines the acceleration is $W^{(2)} / M$, where $M$ is the (inertial) mass of the nucleus. It is convenient to express $M$ in atomic mass units (amu) so that for any nucleus, 


$$
M=\mu(1 \mathrm{amu})
$$

$W^{(2)} / M$ can then be written in the form

$$
\frac{W^{(2)}}{M}=1.4 \times 10^{-16} \frac{N(N-1)}{B \mu} \xi(B)
$$

If $\eta_{\nu \bar{\nu}}$ denotes the constant in Eq. (1.1) corresponding to the $\nu \bar{\nu}$-exchange energy $W^{(2)}$, then from Eq. (1.2)

$$
\frac{\Delta a}{g} \equiv \frac{a_{1}-a_{2}}{g}=\eta_{\nu \bar{\nu}}\left(1.4 \times 10^{-16}\right)\left[\frac{N_{1}\left(N_{1}-1\right)}{B_{1} \mu_{1}} \xi\left(B_{1}\right)-\frac{N_{2}\left(N_{2}-1\right)}{B_{2} \mu_{2}} \xi\left(B_{2}\right)\right] .
$$

In Table I we present the values of the "neutrino charge" $N(N-1) \xi(B) / B \mu$ for the first 92 elements in the Periodic Table, and a plot of this charge is shown in Fig. 3. Following the discussion in Ref. [7] we can show that for an element having several isotopes, with relative abundances $r_{k}\left(\sum_{k} r_{k}=1\right)$, the effective "neutrino charge" is

$$
\frac{N(N-1)}{B \mu} \xi(B) \rightarrow \frac{\sum_{k} r_{k}[N(N-1) \xi(B) / B]_{k}}{\sum_{k} r_{k} \mu_{k}}
$$

Eq. (3.19) was used in obtaining Table $I$.

The sensitivity of the proposed STEP experiment to $\nu \bar{\nu}$-exchange can be estimated by considering as an example the samples used in the experiment of Roll, Krotkov, and Dicke [10] which were $\mathrm{Al}$ and $\mathrm{Au}$ :

$$
\begin{gathered}
{ }_{13} \mathrm{Al}^{27} \Rightarrow \frac{N(N-1)}{B \mu} \xi(B)=0.204 \\
{ }_{79} \mathrm{Au}^{179} \Rightarrow \frac{N(N-1)}{B \mu} \xi(B)=0.321 .
\end{gathered}
$$

Combining Eqs. (3.18) and (3.20) we then have

$$
\frac{\Delta a}{g}=1.7 \times 10^{-17} \eta_{\nu \bar{\nu}}
$$

Since the proposed STEP experiment is designed to achieve a sensitivity $\Delta a / g \simeq 10^{-17}[8]$, it follows from Eq. (3.21) that STEP will be able to set a nontrivial limit on $\eta_{\nu \bar{\nu}}$, 


$$
\left|\eta_{\nu_{i} \bar{\nu}_{i}}\right| \lesssim 0.60
$$

for each neutrino species $i$. If there is a universal breakdown of the WEP for all neutrinos, characterized by a common value of $\eta_{\nu \bar{\nu}}$, then in this case the indicated level of sensitivity in the STEP experiment would lead to

$$
\left|\eta_{\nu \bar{\nu}}\right| \lesssim 0.20
$$

Given a limit such as (3.22) or (3.23), one can in principle work backwards in any detailed theory of WEP violation to infer a constraint on the anomalous coupling of gravity to any of the neutrinos $\nu_{e}, \bar{\nu}_{e}, \nu_{\mu}, \bar{\nu}_{\mu}, \nu_{\tau}$, and $\bar{\nu}_{\tau}$.

In Ref. [7] plots are given of other "charges" that have been considered in the literature including $B / \mu, I_{z} / \mu=(N-Z) / \mu, L / \mu(L=$ lepton number $)$, and the charge associated with a model of Lorentz-noninvariance $[17,18]$. The variations of these charges across the periodic table are different from one another and, with the exception of $I_{z} / \mu$, all of these are different from the "neutrino charge" in Eq. (3.17). A comparison of the "neutrino charge" and $I_{z} / \mu$ is given in Fig. 3 from which we see that these charges have similar shapes, although the two graphs are shifted relative to each other. This shift is physically significant, however, since an important characteristic of a coupling to $I_{z}$ is that many sources (and samples) have $I_{z}=0$ [2]. Hence, if an anomalous acceleration difference were seen in the STEP experiment, or in a future terrestrial experiment, then the "neutrino charge" could be distinguished from $I_{z}$ by utilizing samples for which $I_{z}$ was zero but the neutrino charge was not. It follows from this discussion that all of the generalized "charges" which have been proposed as sources for WEP violation could be distinguished from one another by an appropriate choice of test samples and sources. It follows that if a non-zero value of $\Delta a / g$ were observed, then it would be possible to discriminate among possible sources of this effect by comparing the results obtained using appropriate materials. 


\section{Many-Body Effects}

Following Primakoff and Holstein [37] we note that when the interactions of particles (neutrons in the present case) are described by means of static potentials, as in Eq. (2.25), then relativistic invariance requires that all possible many-body interactions be included along with the 2-body interaction. Ordinarily many-body effects are relatively small in both electromagnetic and strong interactions, for reasons originally discussed in Ref. [37]. However, the considerations that apply to these interactions do not necessarily apply to the interactions between neutrons arising from neutrino-exchange, and hence we briefly examine the many-body effects arising from neutrino exchange in more detail.

Since the Schwinger formula in Eq. (2.5) is relativistically covariant, expanding $W$ in powers of $G_{F}$ gives the $k$-body generalization of $V^{(2)}(r)$ in Eq. (2.25), where $k=3,4, \ldots$ The $k$-body potential thus arises from diagrams in which $k$ neutron lines are attached to a closed neutrino loop, as shown in Fig. 4 for $k=4$. In $O\left(G_{F}^{k}\right)$ there are $(k-1) ! / 2$ pairs of topologically distinct diagrams, with each pair comprising the two diagrams with opposite senses of the neutrino loop momentum. It can be shown that for a spherical nucleus there are no contributions from odd values of $k$, and hence the first non-trivial many-body contribution comes from $k=4$. The 4-body potential arising from Fig. 4(a) has been derived by Hartle $[26,27]$ who finds

$$
\begin{aligned}
V^{(4)}\left(\vec{r}_{12}, \vec{r}_{23}, \vec{r}_{34}, \vec{r}_{41}\right)=\left(\frac{G_{F} a_{n}}{\sqrt{2}}\right)^{4} \frac{1}{4 \pi^{5}}\left\{\frac{4 !}{P_{4} S_{4}^{5}}\right. \\
+2 !\left(\vec{\partial}_{12} \cdot \vec{\partial}_{23}+\vec{\partial}_{23} \cdot \vec{\partial}_{34}+\vec{\partial}_{34} \cdot \vec{\partial}_{41}+\vec{\partial}_{12} \cdot \vec{\partial}_{34}+\vec{\partial}_{12} \cdot \vec{\partial}_{41}+\vec{\partial}_{23} \cdot \vec{\partial}_{41}\right) \frac{1}{P_{4} S_{4}^{3}} \\
\left.+\left[\left(\vec{\partial}_{12} \cdot \vec{\partial}_{23}\right)\left(\vec{\partial}_{34} \cdot \vec{\partial}_{41}\right)-\left(\vec{\partial}_{12} \cdot \vec{\partial}_{34}\right)\left(\vec{\partial}_{23} \cdot \vec{\partial}_{41}\right)+\left(\vec{\partial}_{12} \cdot \vec{\partial}_{41}\right)\left(\vec{\partial}_{23} \cdot \vec{\partial}_{34}\right)\right] \frac{1}{P_{4} S_{4}}\right\}
\end{aligned}
$$

Here $\vec{x}_{1}, \vec{x}_{2}, \vec{x}_{3}$, and $\vec{x}_{4}$ are the coordinates of the 4 particles, with $r_{12}=\left|\vec{x}_{1}-\vec{x}_{2}\right|$, etc., and

$$
\begin{aligned}
& S_{4}=r_{12}+r_{23}+r_{34}+r_{41}, \\
& P_{4}=r_{12} r_{23} r_{34} r_{41},
\end{aligned}
$$


The contributions from diagrams $4(\mathrm{~b})$ and $4(\mathrm{c})$ can be written down immediately by appropriately relabeling the variables in Eq. (3.24).

To determine whether $V^{(4)}$ makes a significant contribution to the weak energy $W$, which is a sum of all the $k$-body contributions, we have integrated Eq. (3.24) numerically [38], and find

$$
U^{(4)}=\frac{4}{\pi R}\left(\frac{G_{F} a_{n}}{2 \pi \sqrt{2} R^{2}}\right)^{4}(7.7)
$$

The notation $U^{(4)}$ indicates that the result in Eq. (3.26) represents the energy of a specific set of 4 particles. We note that, in contrast to the 2-body case, the $k$-body results for $k \geq 4$ are well-behaved even if $r_{c} \rightarrow 0$, and hence we have set $r_{c}=0$. Since these 4 particles can be chosen from among $N$ neutrons in $\left(\begin{array}{c}N \\ 4\end{array}\right)$ ways, where $\left(\begin{array}{c}N \\ k\end{array}\right)$ is the binomial coefficient

$$
\left(\begin{array}{l}
N \\
k
\end{array}\right)=\frac{N !}{k !(N-k) !}
$$

it follows that the 4-body contribution $W^{(4)}$ is given by

$$
\begin{aligned}
W^{(4)} & =U^{(4)}\left(\begin{array}{l}
N \\
4
\end{array}\right) \\
& =(7.7) \frac{4}{\pi R}\left(\frac{G_{F} a_{n}}{2 \pi \sqrt{2} R^{2}}\right)^{4}\left(\begin{array}{c}
N \\
4
\end{array}\right) \\
& =(7.7) \frac{4}{\pi R}\left(\frac{G_{F} a_{n}}{2 \pi \sqrt{2} R^{2}}\right)^{4} \frac{N(N-1)(N-2)(N-3)}{4 !} .
\end{aligned}
$$

For a nucleus with $N \gg 3, W^{(4)}$ can thus be approximated by

$$
W^{(4)} \simeq(7.7) \frac{4 \hbar c}{\pi R}\left[\frac{\left(G_{F} / \hbar c\right) a_{n} N}{2 \pi \sqrt{2} R^{2}}\right]^{4} \frac{1}{4 !},
$$

where factors of $\hbar c$ have been reinstated. From Eq. (3.13) we then find (using the exact expression in Eq. (3.28))

$$
\left[\frac{\left(G_{F} / \hbar c\right) a_{n} N}{2 \pi \sqrt{2} R^{2}}\right]^{4}= \begin{cases}4.5 \times 10^{-31}, & { }_{13} \mathrm{Al}^{27} \\ 1.1 \times 10^{-29}, & { }_{79} \mathrm{Au}^{197}\end{cases}
$$

and 


$$
W^{(4)}=\left\{\begin{array}{l}
6.0 \times 10^{-24} \mathrm{eV},{ }_{13} \mathrm{Al}^{27} \\
1.3 \times 10^{-22} \mathrm{eV},{ }_{79} \mathrm{Au}^{197}
\end{array}\right.
$$

We see from Eqs. (3.15) and (3.31) that $\left|W^{(4)}\right| \ll\left|W^{(2)}\right|$, so that the 4-body contribution to the weak energy is indeed negligible. For $k>4$ the $k$-body contribution $W^{(k)}$ will be of order

$$
W^{(k)} \sim\left(\frac{\hbar c}{R}\right)\left(\frac{G_{F} a_{n} N}{R^{2}}\right)^{k}
$$

and from Eq. (3.30) it follows that higher order many-body effects make increasingly smaller contributions to $W$. We thus conclude that the neutrino-exchange energy of a nucleus is dominated by the 2-body contribution given in Eq. (3.9).

We note in passing that the preceding formalism can be applied directly to a neutron star, which can be approximated for present purposes as a large nucleus. Consider, for example, the observed pulsar in the Hulse-Taylor binary system PSR $1913+16$ [39-41]. The mass $M_{1}$ of this pulsar is accurately known $[40,41]$,

$$
M_{1}=1.4411(7) M_{\odot}
$$

and hence the mass of a typical neutron star can be taken to be

$$
M=1.4 M_{\odot}=2.8 \times 10^{33} \mathrm{~g}
$$

To calculate the number of neutrons $N$ we can ignore the contribution to $M$ from gravitational binding energy, and assume that the neutron star is composed exclusively of neutrons. Using Eq. (3.34) then leads to

$$
N=1.7 \times 10^{57}
$$

The radius $R$ of the neutron star, although not directly observable, can be inferred in various models. We assume the nominal value $R=10 \mathrm{~km} \equiv R_{10}$ which corresponds to a mass density $\rho_{m}$ and a number density $\rho$ given by 


$$
\begin{gathered}
\rho_{m}=6.7 \times 10^{14} \mathrm{~g} \mathrm{~cm}^{-3}, \\
\rho=4.0 \times 10^{38} \mathrm{~cm}^{-3} .
\end{gathered}
$$

These values of $N, R$, and $\rho$ are typical of the results that arise in existing models of neutrons stars $[42,43]$. We then have

$$
\frac{\left(G_{F} / \hbar c\right) N}{R_{10}^{2}}=7.6 \times 10^{12}
$$

It follows from Eq. (3.38) that for a neutron star higher-order many-body effects make increasingly larger contributions to $W$, in contrast to what we found above for nuclei. Using Eqs. (3.32) and (3.38) it is straightforward to show that successive terms in the expansion of the Schwinger formula in Eq. (2.5) increase in magnitude, and that $W^{(8)}$ would exceed the known mass-energy of the pulsar. Although this may indicate a breakdown of perturbation theory, an alternative possibility is that perturbation theory remains valid but that neutrinos have a small mass $m_{\nu}$. In such a case the $\nu \bar{\nu}$-exchange force "saturates" and, for an appropriate value of $m_{\nu}$, the mass-energy of the pulsar arising from neutrino-exchange would be reduced to a physically acceptable value. The critical value of $m_{\nu}$ (for any species $\nu_{e}, \nu_{\mu}$, or $\left.\nu_{\tau}\right)$ is

$$
m_{\nu} c^{2} \gtrsim \frac{2}{3 e^{3}} \frac{G_{F}}{\sqrt{2}}\left|a_{n}\right| \rho=0.4 \mathrm{eV}
$$

where now $\ln e=1$. Interestingly, for $\nu_{e}$ this is just below the existing upper bound, $m_{\nu_{e}} \lesssim 7 \mathrm{eV}[21]$. A more detailed discussion of these calculations will be presented elsewhere, along with similar results for white dwarfs where the same problem also arises.

\section{DISCUSSION AND SUMMARY}

The object of this paper has been to study for the first time the 2-body $\nu \bar{\nu}$-exchange contribution $W^{(2)}$ to the mass-energy of a nucleus. We have shown that because this is a longrange interaction, $W^{(2)}$ can be calculated semiclassically, just as in the case of the Coulomb 
energy. Although the result in Eq. (3.15), like the Coloumb result, applies strictly to spherical nuclei, both results should be good approximations for most nuclei. The numerical results for $W^{(2)} / M$ and $\Delta a / g$ in Eqs. (3.17) and (3.21) are interesting because they indicate that at the level of sensitivity of the proposed STEP experiment $\left(\Delta a / g \simeq 10^{-17}\right)$, a non-trivial constraint on the WEP-violating parameter $\eta_{\nu \bar{\nu}}$ could be set. Were an anomaly to be seen in the STEP experiment, then the characteristic composition dependence of the $\nu \bar{\nu}$-exchange contribution as shown in Fig. 3 could be used to test whether this contribution or some other was the source of the WEP violation.

We note that both the magnitude and composition dependence of the $\nu \bar{\nu}$-exchange force are consequences of the fact that the interaction mediated by $\nu \bar{\nu}$ is long-ranged. This uniquely distinguishes $\nu \bar{\nu}$-exchange from other weak interaction contributions: The longrange is what is responsible for the combinatoric factor $\sim N(N-1) / 2$, and because all the neutron contributions thus add coherently, this process likely constitutes the dominant weak effect in $O\left(G_{F}^{2}\right)$. The long-range nature of this interaction is also what allows a detailed semi-classical calculation to be carried out, in analogy to the Coulomb energy of the nucleus.

Many-body neutrino-exchange contributions have also been investigated to verify that these would not alter the conclusions to be drawn from $W^{(2)}$. We have shown that the many-body contributions are indeed small in all nuclei, although they could be quite large in a neutron star. Interestingly this observation could lead to a lower bound on neutrino masses.

\section{ACKNOWLEDGMENTS}

One of the authors (E. F.) wishes to thank Jim Hartle for making available his unpublished notes on neutrino exchange, and for his permission to reproduce parts of these notes in Sec. II. We wish to thank Shu-Ju Tu for help in carrying out the numerical work, and we are indebted to Francis Everitt, Mark Haugan, and Paul Worden for many informative discussions concerning the STEP experiment. This work is supported by the U.S. Department 
of Energy under Contract No. DE-AC02-76ER01428. 


\section{REFERENCES}

[1] E.G. Adelberger, B.R. Heckel, C.W. Stubbs, and W.F. Rogers, Annu. Rev. Nucl. and Part. Sci. 41, 269 (1991).

[2] E. Fischbach and C. Talmadge, Nature 356, 207 (1992).

[3] E. Fischbach, G.T. Gillies, D.E. Krause, J.G. Schwan and C. Talmadge, Metrologia 29, 215 (1992).

[4] Y. Fujii, Int. J. Mod. Phys. A 6, 3505 (1991).

[5] C. M. Will, Theory and Experiment in Gravitational Physics, Revised Edition (Cambridge University Press, Cambridge, 1993).

[6] E. Fischbach, D. Sudarsky, A. Szafer, C. Talmadge, and S.H. Aronson, Phys. Rev. Lett. 56, $3(1986)$.

[7] E. Fischbach, D. Sudarsky, A. Szafer, C. Talmadge, and S.H. Aronson, Ann. of Phys. (NY) 182, 1 (1988).

[8] Proceedings of the STEP Symposium, Pisa, Italy, 6-8 April, 1993 (to be published).

[9] R. v. Eötvös, D. Pekár, and E. Fekete, Ann. Phys. (Leipzig) 68, 11 (1922); Roland Eötvös Gesammelte Arbeiten, edited by P. Selényi (Akadémiai Kiado, Budapest, 1953), pp. $307-372$.

[10] P.G. Roll, R.V. Krotkov, and R.H. Dicke, Ann. of Phys. (NY) 26, 442 (1964).

[11] A.H. Wapstra and G.J. Nijgh, Physica 21, 796 (1955).

[12] A. Bohr and B. R. Mottelson, Nuclear Structure, Vol. 1, (Benjamin, New York, 1969), p. 141.

[13] S. de Benedetti, Nuclear Interactions (Wiley, New York, 1964), p. 73.

[14] M.G. Bowler, Gravitation and Relativity, (Pergamon, Oxford, 1976) p. 27ff. 
[15] K. Nordtvedt, Jr., Science 178, 1157 (1972).

[16] M.P. Haugan and C.M. Will, Phys. Rev. Lett. 37, 1 (1976).

[17] E. Fischbach, M.P. Haugan, D. Tadić, and H.-Y. Cheng, Phys. Rev. D32, 154 (1985).

[18] H.B. Nielsen and I. Picek, Nucl. Phys. B211, 269 (1983).

[19] L.I. Schiff, Phys. Rev. Lett. 1, 254 (1958); Proc. Nat. Acad. Sci. 45, 69 (1959).

[20] T. Goldman, R.J. Hughes, and M.M. Nieto, Phys. Rev. D36, 1254 (1987), and Sci. Am. 258, No. 3, 48 (1988).

[21] Particle Data Group (L. Montanet, et al.), Phys. Rev. D 50, 1173 (1994).

[22] G. Feinberg and J. Sucher, Phys. Rev. 166, 1638 (1968).

[23] G. Feinberg, J. Sucher, and C. -K. Au, Phys. Rep. 180, 83 (1989).

[24] S. D. H. Hsu and P. Sikivie, Phys. Rev. D 49, 4951 (1994).

[25] J. Schwinger, Phys. Rev. 94, 1362 (1954).

[26] J. B. Hartle, Phys. Rev. D 1, 394 (1970); some minor misprints in this reference have been corrected here.

[27] J. B. Hartle (unpublished).

[28] E. Fermi, Nuclear Physics, Revised Edition (University of Chicago, Chicago, 1950), p. $112 \mathrm{ff}$.

[29] D. Lurié, Particles and Fields (Wiley-Interscience, New York, 1968).

[30] B. deWit and J. Smith, Field Theory in Particle Physics (North-Holland, Amsterdam, 1986).

[31] J. J. Sakurai, Advanced Quantum Mechanics (Addison-Wesley, Reading, 1967).

[32] L. A. Santaló, Integral Geometry and Geometric Probability (Addison-Wesley, Reading, 
1976), p. 212.

[33] M. G. Kendall and P. A. P. Moran, Geometrical Probability (Hafner, New York, 1963), p. 53.

[34] A. W. Overhauser (unpublished).

[35] A. Bohr and B. R. Mottelson, op. cit., p. 267.

[36] A. Bohr and B. R. Mottelson, op. cit., p. 192.

[37] H. Primakoff and T. Holstein, Phys. Rev. 55, 1218 (1939).

[38] S. -J. Tu and E. Fischbach (unpublished).

[39] R. A. Hulse and J. H. Taylor, Astrophys. J. Lett. 195, L51 (1975).

[40] J. H. Taylor and J. M. Weisberg, Astrophys. J. 345, 434 (1989).

[41] C. M. Will, Nature 355, 111 (1992).

[42] S. L. Shapiro and S. A. Teukolsky, Black Holes, White Dwarfs, and Neutron Stars (Wiley-Interscience, New York, 1983), p. 251.

[43] M. A. Alpar, in Timing Neutron Stars, edited by H. Ögelman and E. P. J. van den Heuvel (Klüwer, Dordrecht, 1989), p. 431. 


\section{TABLES}

TABLE I. Average value of the "neutrino charge" $N(N-1) \xi(B) / B \mu$ for the first 92 elements of the Periodic Table. See text for further details.

\begin{tabular}{|c|c|c|c|c|c|}
\hline Element & $N(N-1) \xi(B) / B \mu$ & Element & $N(N-1) \xi(B) / B \mu$ & Element & $N(N-1) \xi(B) / B \mu$ \\
\hline Hydrogen & 0.000 & Germanium & 0.265 & Europium & 0.303 \\
\hline Helium & 0.084 & Arsenic & 0.265 & Gadolinium & 0.311 \\
\hline Lithium & 0.172 & Selenium & 0.276 & Terbium & 0.309 \\
\hline Beryllium & 0.183 & Bromine & 0.269 & Dysprosium & 0.313 \\
\hline Boron & 0.181 & Krypton & 0.278 & Holmium & 0.313 \\
\hline Carbon & 0.159 & Rubidium & 0.275 & Erbium & 0.313 \\
\hline Nitrogen & 0.166 & Strontium & 0.275 & Thulium & 0.311 \\
\hline Oxygen & 0.171 & Yttrium & 0.270 & Ytterbium & 0.315 \\
\hline Fluorine & 0.198 & Zirconium & 0.271 & Lutetium & 0.314 \\
\hline Neon & 0.183 & Niobium & 0.268 & Hafnium & 0.317 \\
\hline Sodium & 0.201 & Molybdenum & 0.272 & Tantalum & 0.317 \\
\hline Magnesium & 0.191 & Technetium $^{\mathbf{a}}$ & 0.271 & Tungsten & 0.318 \\
\hline Aluminum & 0.204 & Ruthenium & 0.276 & Rhenium & 0.318 \\
\hline Silicon & 0.192 & Rhodium & 0.274 & Osmium & 0.322 \\
\hline Phosphorus & 0.206 & Palladium & 0.280 & Iridium & 0.321 \\
\hline Sulfur & 0.195 & Silver & 0.276 & Platinum & 0.322 \\
\hline Chlorine & 0.213 & Cadmium & 0.286 & Gold & 0.321 \\
\hline Argon & 0.242 & Indium & 0.286 & Mercury & 0.323 \\
\hline Potassium & 0.210 & Tin & 0.293 & Thallium & 0.326 \\
\hline Calcium & 0.200 & Antimony & 0.295 & Lead & 0.327 \\
\hline Scandium & 0.230 & Tellurium & 0.308 & Bismuth & 0.326 \\
\hline Titanium & 0.238 & Iodine & 0.297 & Polonium $^{\mathrm{a}}$ & 0.321 \\
\hline
\end{tabular}




\begin{tabular}{llllll} 
Vanadium & 0.247 & Xenon & 0.304 & Astatine $^{\mathrm{a}}$ & 0.318 \\
Chromium & 0.238 & Cesium & 0.302 & Radon $^{\mathrm{a}}$ & 0.337 \\
Manganese & 0.245 & Barium & 0.309 & Francium $^{\mathrm{a}}$ & 0.333 \\
Iron & 0.236 & Lanthanum & 0.306 & Radium $^{\mathrm{a}}$ & 0.335 \\
Cobalt & 0.244 & Cerium & 0.302 & Actinium $^{\mathrm{a}}$ & 0.332 \\
Nickel & 0.227 & Praseodymium & 0.298 & Thorium $^{\mathrm{a}}$ & 0.337 \\
Copper & 0.247 & Neodymium & 0.301 & Protactinium $^{\mathrm{a}}$ & 0.331 \\
Zinc & 0.246 & Promethium & \\
Gallium & 0.260 & Samarium & 0.296 & Uranium $^{\mathrm{a}}$ & 0.339 \\
\hline \hline
\end{tabular}

${ }^{a}$ No stable isotopes. 


\section{FIGURES}

FIG. 1. Coupling of gravity to $e^{+}-e^{-}$pairs in the field of a nucleus. The solid dots $(\bullet)$ denote the electromagnetic interaction.

FIG. 2. Interaction of two neutrons via neutrino-antineutrino $(\nu \bar{\nu})$ exchange. The heavy (light) lines denote neutrons (neutrinos).

FIG. 3. Plot of the average "neutrino charge" $N(N-1) \xi(B) / B \mu$ and $I_{z} / \mu$ as functions of atomic number $Z$ for the first 92 elements. Although both plots show a similar variation, these charges can be distinguished from each other by noting that $I_{z} / \mu=0$ for several elements and various compounds. See text for details.

FIG. 4. Contributions to the 4-body potential energy arising from neutrino exchange. As before, heavy (light) lines denote neutrons (neutrinos). Each of the diagrams (a), (b), and (c) is topologically different from the others, as can be seen by redrawing the graphs as shown. For each of these diagrams there is another that is obtained by reversing the sense of the neutrino loop momentum. 


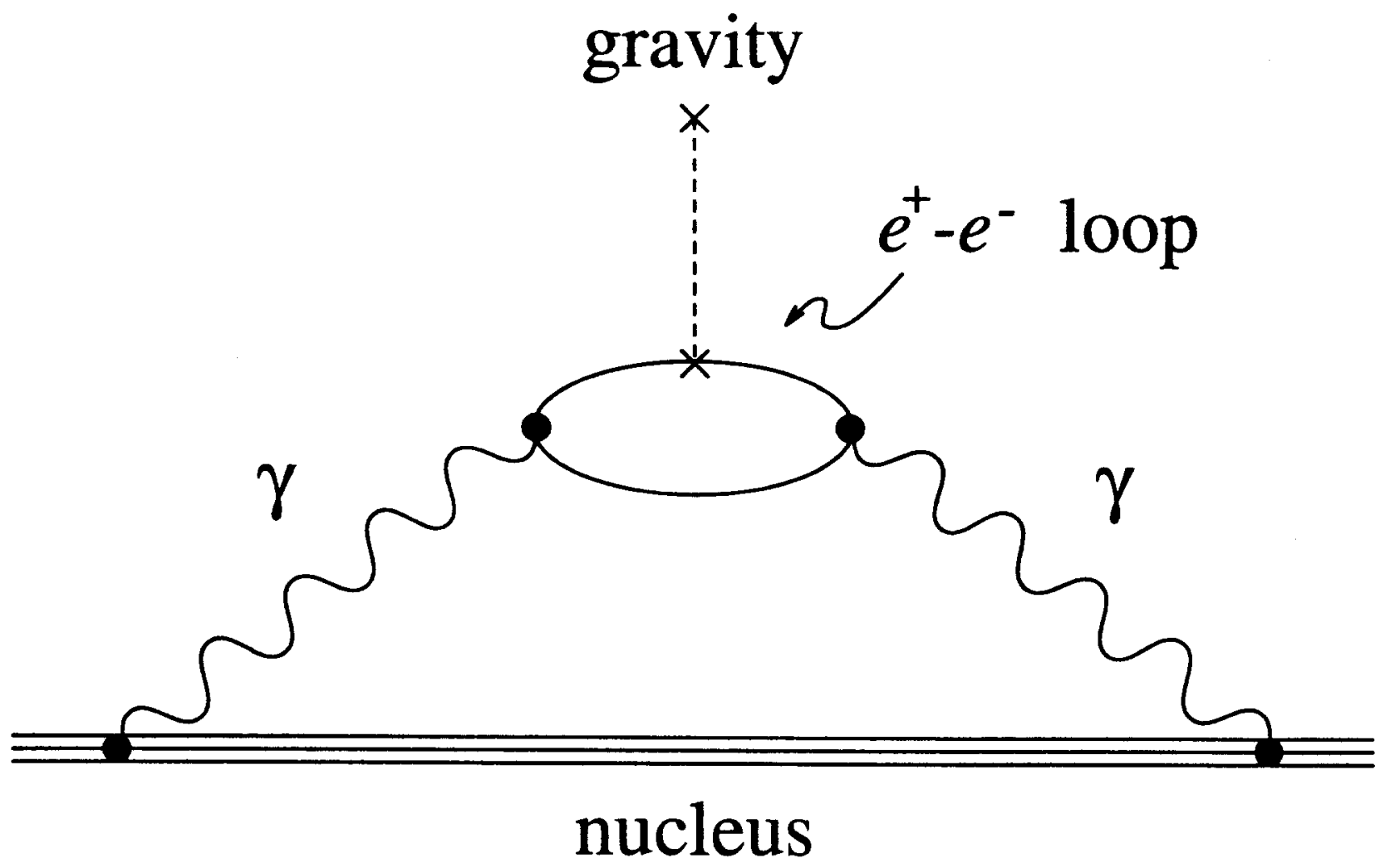




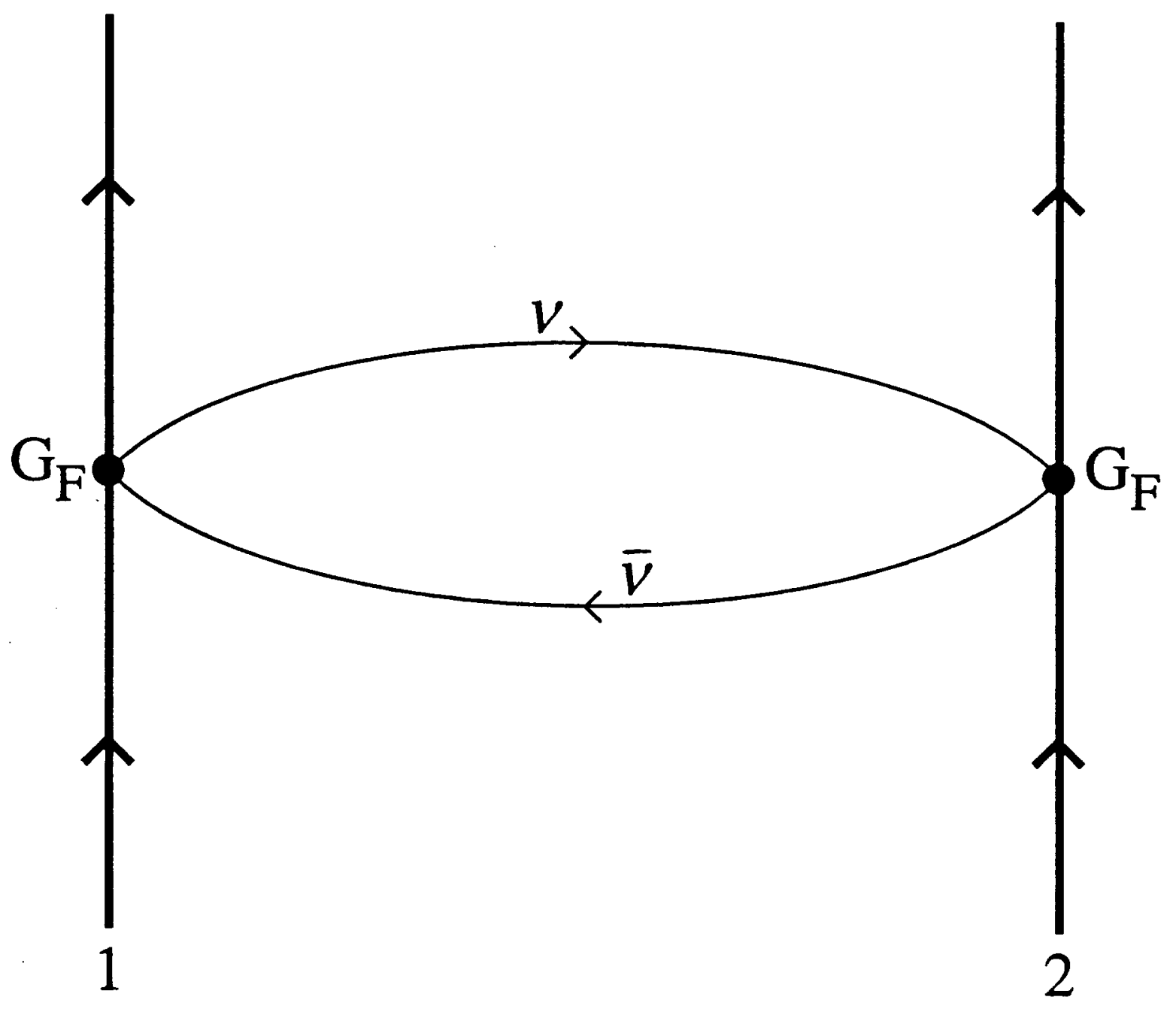




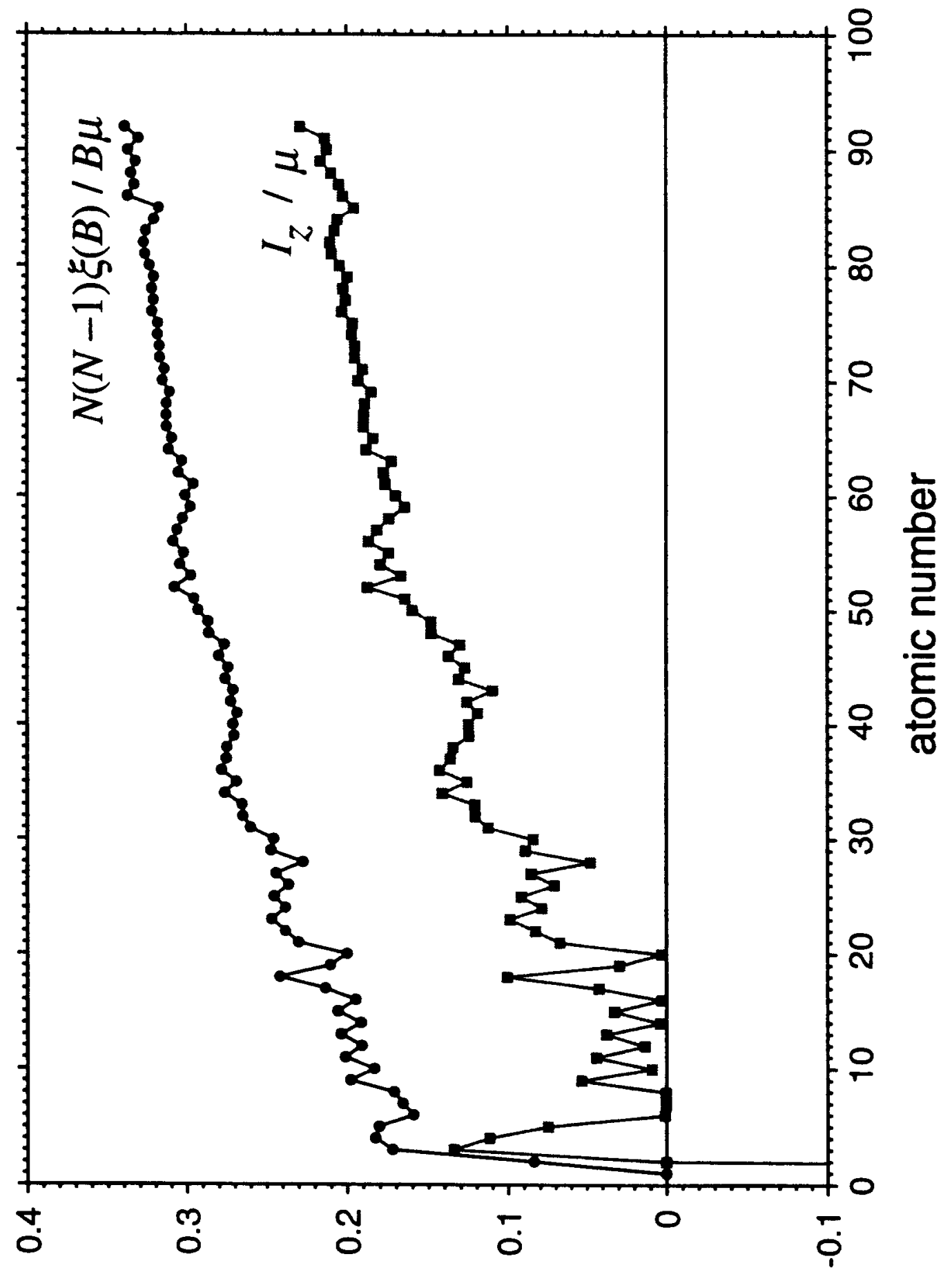


(a)
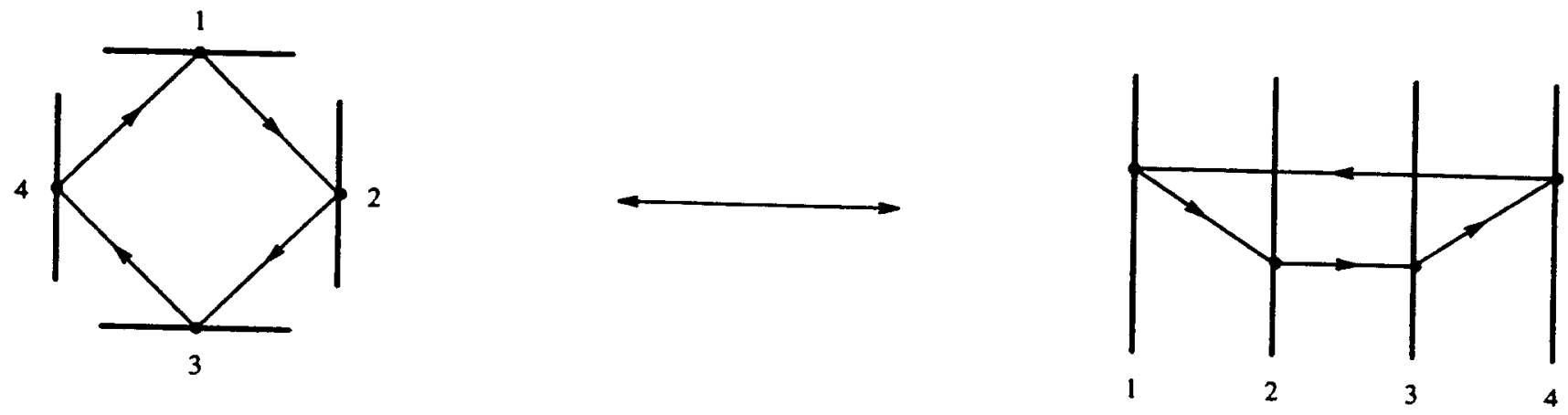

(b)
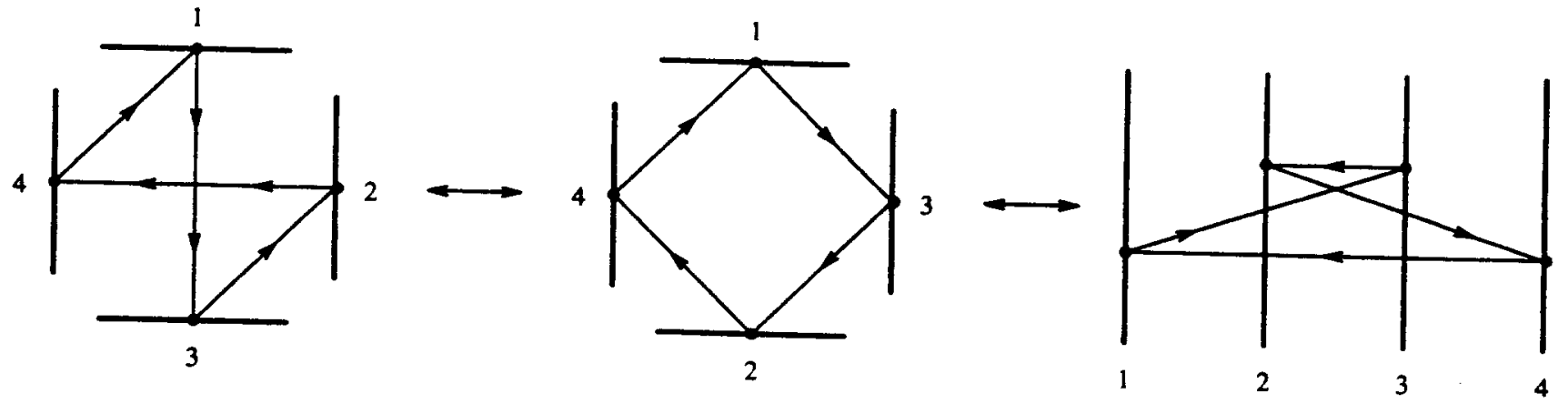

(c)
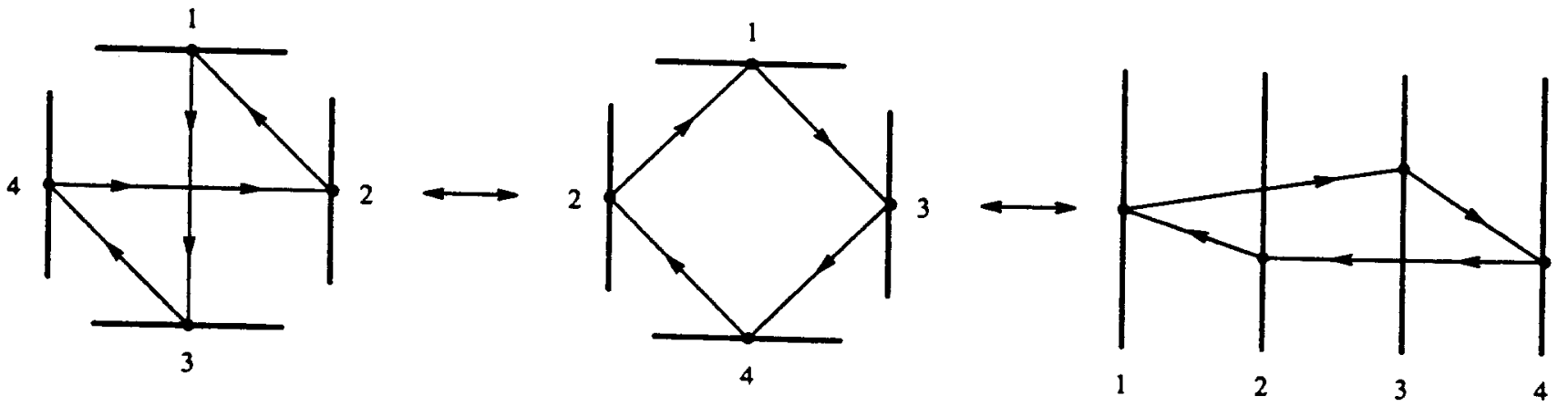
